\title{
Roles of the ovarian surface epithelium in ovulation and carcinogenesis
}

\author{
William J. Murdoch and Anna C. McDonnel \\ Reproductive Biology Program, University of Wyoming, Laramie, WY 82071, USA
}

\begin{abstract}
Although ovarian mechanisms of ovulation have been a subject of investigation for more than a century, essential regulatory pathways remain uncertain. A role for the ovarian surface epithelium in ovulation has recently been demonstrated. Ovarian surface epithelial cells in close contact with the apical wall of preovulatory ovine follicles secrete a urokinasetype plasminogen activator in response to surge concentrations of (locally delivered) gonadotrophins. Urokinase activates latent collagenases and stimulates release of tumour necrosis factor $\alpha$ from thecal endothelium. Tumour necrosis factor $\alpha$ progressively induces matrix metalloproteinase gene expression, apoptosis and inflammatory necrosis. Collagenolysis and cellular death are a prelude to stigma formation and ovarian rupture. Epithelium exfoliated from the dome of ovulatory follicles is replenished by generative stem cell replication and migration from the wound edges. Common epithelial ovarian cancer has been related to successive bouts of ovulation and mitosis. The integrity of the DNA of surface cells circumjacent to the ovarian rupture site is compromised during the ovulatory process. Clonal expansion of an epithelial cell with damaged (unrepaired) DNA is a putative factor in carcinogenesis. Ovarian cancer is a deadly insidious disease because typically it is asymptomatic until the malignancy has reached beyond the ovaries.
\end{abstract}

The mammalian ovary is covered by a singular layer of epithelial cells. The rather unassuming character of the ovarian surface epithelium has generally inclined reproductive physiologists to question its functional significance. However, recent experimental evidence indicates that the ovarian epithelium is an obligate component of the ovulatory process. That most ovarian cancers arise by transformation of a surface epithelial cell affected by ovulation has been a point of theory for three decades. The objectives of this overview are to consider the morphology and developmental biology of the ovarian surface epithelium and to delineate its apparent roles in ovulation and pathogenesis.

\section{Anatomy and embryonic origin of the ovarian surface epithelium}

Ovarian surface epithelial cells vary in type from simple squamous to cuboidal to low pseudostratified columnar. The surface epithelium is supported over the ovarian cortical interstitium (tunica albuginea) by a basement membrane (Fig. 1) and is held together laterally by desmosomes and gap or tight junctional complexes. Surface cells are continuous at the hilum with the mesothelium of the ovarian ligament (mesovarium) and peritoneum. Preferential outgrowth of a

Email:wmurdoch@uwyo.edu preovulatory follicle brings it into close apposition with the ovarian surface. In most mammals, the entire surface of the ovary, other than those regions disrupted by ovulation, is covered by epithelial cells. However, in equids, the ovarian epithelium (and ovulation) is restricted to a discrete area of depression known as the fossa, and the remainder of the ovary is encased by serosa-containing elastic bands of connective tissue (Walt et al., 1979).

Ovarian surface epithelial cells have a mesodermal derivation shared with the epithelia of the urogenital system and adrenal cortex. Mesoderm segregates during embryonic development into pluripotent mesenchyme and coelomic epithelium (peritoneal mesothelium). Mullerian mesothelium is the precursor of oviductal, endometrial, and cervical epithelia. Ovarian surface epithelium differentiates after invagination of the coelomic mesothelium over the gonadal ridges (Byskov, 1986). In species with an ovulation fossa, the cortex (with modified epithelium) migrates into the medullary portion of the ovary during the postnatal period (Walt et al., 1979). Early investigators assumed that ova were derived from the ovarian surface; hence, the misnomer 'germinal' epithelium.

\section{Role of the ovarian surface epithelium in the mechanism of ovulatory follicular rupture}

The contention that ovarian surface epithelial cells participate actively in the biomechanics of gonadotrophin-induced 


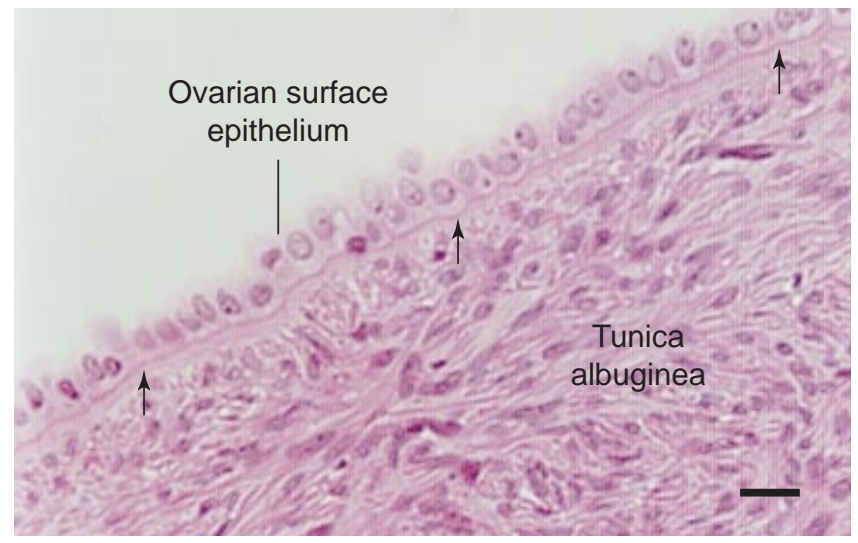

Fig. 1. Light photomicrograph of an ovine ovarian surface epithelium in cross-section. Healthy cells contain a large nucleus with a prominent nucleolus. Arrows indicate the basal lamina. Scale bar represents $10 \mu \mathrm{m}$.

ovulatory follicular rupture was initially supported by circumstantial anatomical evidence. Several studies indicated that connective tissue degeneration begins at the ovarian surface and advances toward the follicular wall. Proteolytic enzymes released from cytoplasmic granules of epithelial cells appeared to degrade the tunica albuginea and underlying theca, thereby weakening the apical follicular wall (Bjersing and Cajander, 1975).

The notion that the ovarian epithelium has a function in the ovulatory mechanism was discounted when Rawson and Espey (1977) reported that ovulation still occurred from some rabbit follicles in which the surfaces had been scraped. However, the efficacy of surface epithelial removal was not confirmed (and numbers of ovulatory ovarian rupture points were reduced by scraping). Ovulation in frogs in vitro (Schuetz and Lessman, 1982) and sheep in vivo (Colgin and Murdoch, 1997) is blocked by surgical removal of the ovarian surface epithelium. Merely handling the surface of ovaries (which disrupts the surface epithelium) inhibits ovulation in pigs (Hall et al., 1993).

Collagen breakdown and cellular death (apoptosis and inflammatory necrosis) within the apex of the preovulatory ovine follicle are hallmarks of impending ovulation. An integrative model is presented in which gonadotrophic stimulation of plasminogen activator (PA) secretion by ovarian surface epithelial cells contiguous with the preovulatory follicle elicits a localized increase in tissue plasmin, which activates latent collagenases and releases tumour necrosis factor $\alpha$ (TNF- $\alpha$ ) from its anchor along the thecal endothelium. Soluble TNF- $\alpha$ potentiates collagenolysis by induction of gene expression of matrix metalloproteinase 1 (MMP-1) and MMP-2 and, at relatively higher concentrations, mediates epithelial-vascular dissipation. Consequent weakening of the apical follicular wall leads to ovarian stigma formation and ovulatory rupture (Fig. 2).
Gonadotrophic upregulation of plasmin at the ovarian surface-preovulatory follicular interface

Plasmin (fibrinolysin) is a pleiotrophic serine protease that is derived from the zymogen plasminogen by enzymatic activation. Two forms of PA have been characterized in vertebrates: urokinase $(\mathrm{u})$ and tissue $(\mathrm{t})$ types. Most functional studies indicate that UPA mediates tissue degradation, whereas IPA (which has a strong affinity for fibrin) modulates thrombolysis (Danø et al., 1985).

An increase in plasmin biosynthesis within the apical hemisphere and conjoined tunica albuginea of preovulatory follicles in ewes has been attributed to secretion of UPA by ovarian surface epithelial cells (tPA was undetectable). When ovarian surface epithelium was removed, the follicular increase in uPA was negated (Colgin and Murdoch, 1997). Furthermore, ovulation was suppressed by intrafollicular injection of UPA (but not tPA) antibodies (Colgin and Murdoch, 1997) or $\alpha_{2}$-antiplasmin (Murdoch, 1998a). In addition, plasminogen activators were increased preferentially within the apices of preovulatory pig (Smokovitis et al., 1988) and rat (Peng et al., 1993) follicles. Intrabursal administration of inhibitors of the PA-plasmin system decreased ovulation rates in rats (Tsafriri and Reich, 1991).

Sheep ovarian surface epithelial cells secrete uPA in a basal direction (that is, towards the tunica albuginea and apical follicular wall) in response to $\mathrm{LH}$. Receptors for $\mathrm{LH}$ on ovarian surface cells are upregulated at pro-oestrus by oestradiol of preovulatory follicular origin (Murdoch et al., 1999a). Ovarian epithelium in close proximity to the preovulatory follicle is readily exposed to surge concentrations of LH owing to an acute increase in permeability of the thecal vascular wreath (Cavender and Murdoch, 1988). Secretion of PAs by gonadotrophin-stimulated thecal and granulosal cells of rodent follicles has been established; both UPA and tPA contribute to the efficiency of ovulation, although ovulatory rates are not altered in mice with a single gene deficiency (Hägglund et al., 1996).

\section{Plasmin-induced endothelial secretion of TNF- $\alpha$}

The cytokine TNF- $\alpha$ is expressed as an integral transmembrane precursor protein that yields an extracellular domain subunit upon cleavage. Mature (soluble) TNF- $\alpha$ is a noncovalent homotrimer. Plasma membrane glycoprotein receptors for TNF- $\alpha(\mathrm{RI}, \mathrm{RII})$ are present on virtually all nucleated cells, including those of the mammalian ovary (Terranova, 1997).

TNF- $\alpha$ is localized to the thecal endothelial cells of preovulatory ovine follicles. Immunostaining of endothelium within the follicular apex declined abruptly with the approach of ovulation, although cells within the counterpart basal wall were unaffected. The cytokine was secreted, within a limited diffusion radius, into the progenitor site of rupture (Murdoch et al., 1997). Bioactive TNF- $\alpha$ was truncated from endothelial cells by plasmin (Murdoch et al., 1999b). Preovulatory follicles of other species (for example, 


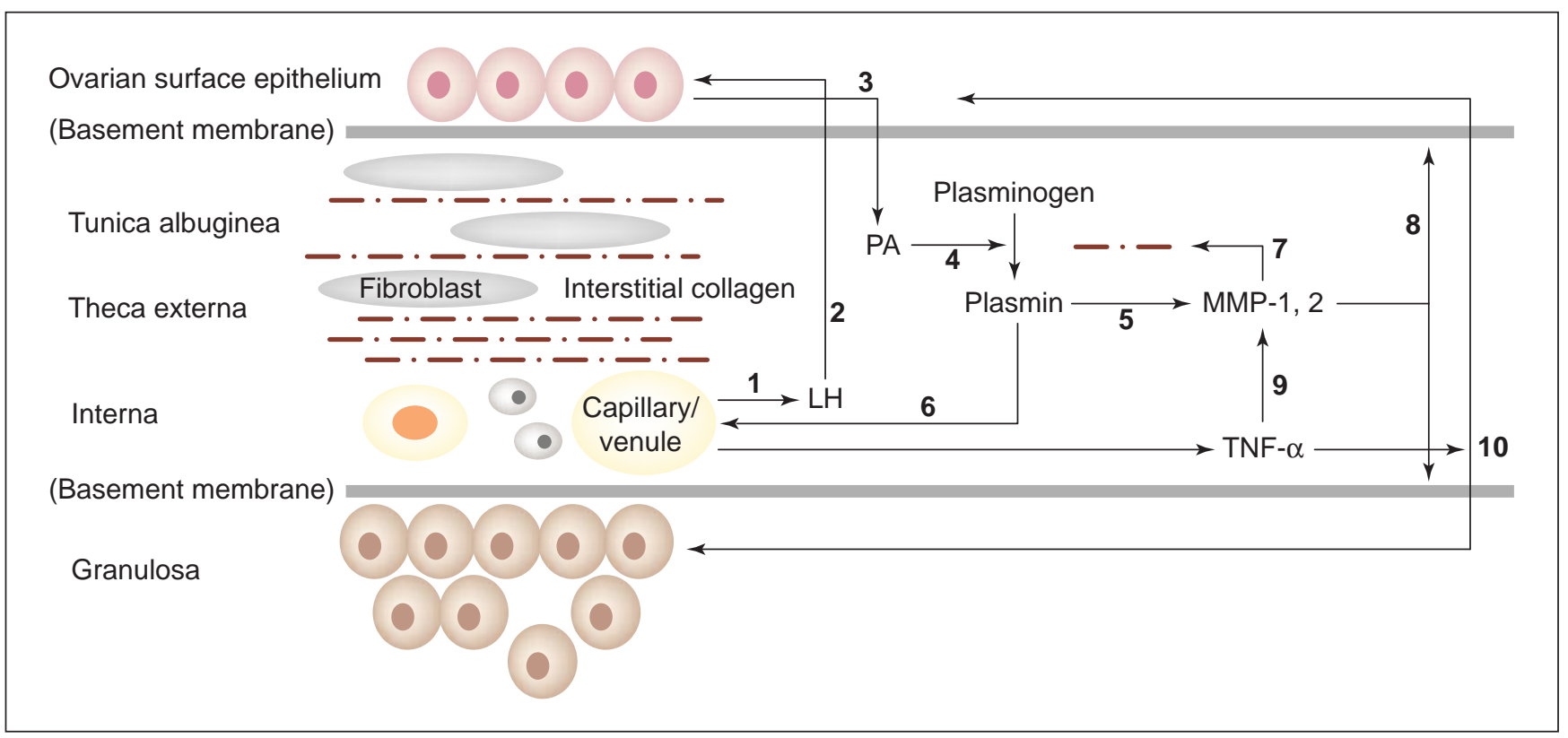

Fig. 2. Synopsis of the interactions of $\mathrm{LH}$, ovarian cell types, plasminogen activator (PA)-plasmin, collagenases, and tumour necrosis factor $\alpha(\mathrm{TNF}-\alpha)$ in the degeneration of the apical follicular wall during the ovulatory process of sheep. (1) The thecal vascular transudate containing LH is (2) delivered to receptor-bearing cells of the ovarian surface epithelium, (3) stimulating secretion of PA. (4) Interstitial plasminogen is converted to plasmin, which (5) activates collagenases and (6) cleaves TNF- $\alpha$ exodomain from endothelial cells. (7) Collagenases disrupt the fibril network of the theca and tunica albuginea (via matrix metalloproteinase 1 (MMP-1) and (8) promote digestion of the basement membranes that support the ovarian and granulosal epithelia (via MMP-2). (9) TNF- $\alpha$ induces MMP production and (10) cellular death; proteolysis and cellular deletion cause progressive thinning of the ovarian wall, stigma formation, and follicular rupture.

rats, cows, humans) secrete TNF- $\alpha$ (Terranova, 1997), although cellular and molecular release processes have not been elucidated.

Target tissue effects of TNF- $\alpha$ are receptor subtype- and concentration-dependent. Receptors bind trimeric ligand through a homologous extracellular amino terminal motif. The cytoplasmic segment of TNFRI contains a death domain that, upon receptor aggregation, can evoke a proteolytic cascade leading to apoptotic (internucleosomal) DNA fragmentation and cellular dissolution. Non-lethal transcriptional events (such as MMP gene expression) also can be activated by TNFRI and TNFRII ligation. It remains unclear which mechanisms dictate the pathway of signal transduction outcome towards genomic stimulation with or without programmed death (Warzocha and Salles, 1998). At high tissue concentrations, TNF- $\alpha$ initiates microvascular coagulation associated with acute inflammation and necrotic cellular death (Larrick and Wright, 1990). The addition of TNF- $\alpha$ to perfusates of rat ovaries enhances ovulation rates elicited by LH (Brännström et al., 1995).

\section{Collagenolysis}

Type I (interstitial) collagen is the primary supportive fabric of the ovarian-follicular wall. Basement membranes that circumscribe thecal capillary beds and on which granulosal cells and ovarian surface epithelium abut are composed of type IV collagen (Luck, 1994). Mammalian collagenases belong to a family of metalloproteinases that truncate each of the polypeptide chains of collagen at sites near the N-terminus; the enzymes share many structural and functional attributes, but differ in substrate specificities (Birkedal-Hansen, 1995). Collagen catabolism is a prerequisite of follicular distension and ovulatory rupture (Woessner et al., 1989).

In preovulatory ovine follicles, there is a direct association of apical plasmin accumulation (Colgin and Murdoch, 1997) with the onset of collagenolysis (Murdoch and McCormick, 1992). Explants of follicular wall release fragments of collagen upon exposure to plasmin, and injection of $\alpha_{2}$-antiplasmin into preovulatory follicles suppresses collagenase bioactivity of tissue extracts (Murdoch, 1998a). Intrafollicular injection of TNF- $\alpha$ antibodies, which prevent ovulation (Murdoch et al., 1997), also inhibit the increase in collagenolysis (Johnson et al., 1999).

Matrix-degrading effects of metalloproteinases are dependent upon de novo production, proteolytic activation, and endogenous tissue inhibitor (TIMP) concentrations. Excision of latent collagenases by plasmin, permitting a second (autolytic) cleavage of the Cys- $\mathrm{Zn}^{2+}$ bond that stabilizes the propeptide, exposes the catalytic domain of the enzyme (Birkedal-Hansen, 1995). An accessory effect of 
TNF- $\alpha$ on follicular MMP-1 and -2 biosyntheses is exerted at transcription (Johnson et al., 1999; Gottsch et al., 2000). Steroidogenic (granulosal-thecal) cells and fibroblasts are sources of MMPs. A co-ordinate increase in production of TIMPs by the granulosal cells of periovulatory follicles serves to limit the extent of tissue destruction, assuring that a viable corpus luteum can be formed (Smith et al., 1999).

\section{Cellular death}

As the time of ovulation approaches in ewes, there is an increase in apoptosis (plasma membrane phosphatidylserine translocation and internucleosomal DNA fragmentation) among cells within the follicle-associated ovarian surface epithelium (that is, after the gonadotrophin-induced release of UPA) and adjacent tunica albuginea and apical follicular wall. The initial apoptotic wave is succeeded by necrosis (indiscriminate DNA degradation and cellular lysis), extravasation of blood cells, and vascular collapse. At the ischaemic site of impending rupture, follicles are essentially devoid of ovarian surface and granulosal epithelia (Murdoch et al., 1999c). The cytotoxic response within the formative ovulation papilla is mediated by TNF- $\alpha$ (Murdoch et al., 1997, 1999b). Apical ovarian cells are safeguarded from destruction by the lipophilic ovulation inhibitor, indomethacin, which abrogates (via a prostaglandinindependent biophysical effect on the plasma membrane) TNF- $\alpha$ signal transmission (Murdoch and Lund, 1999).

The surface of ovulatory sheep ovaries is not completely restored until after luteal involution; in some instances, small nests of epithelial cells become incorporated into the cortical interstitium (Murdoch, 1994). It has been proposed that ovarian inclusion bodies of surface epithelium are normally eliminated via the Fas apoptotic system (Ghahremani et al., 1999).

\section{Common (surface) epithelial ovarian cancer}

Although the surface epithelium represents only a diminutive fraction of the diverse cell types that comprise the ovary, it accounts for over $90 \%$ of all cancers attributed to this complex organ. Recognition of the principal role of the ovarian surface epithelium in malignancy has been credited to Sir Spencer Wells in 1872 (Hamilton, 1992).

\section{Epidemiology}

Ovarian cancer is the fifth most frequent cancer in women (after cancers of the breast, colorectum, lung and endometrium). Ovarian cancer carries a 1-in-70 lifetime risk. Diagnoses of epithelial ovarian cancer increase with age (average age at initial presentation is 61). Most cases are sporadic; 5-10\% are familial. Risk among first degree relatives (mother, sister, daughter) can be as great as 50\%. Incidences are highest in the industrialized cultures of Europe and North America. Circumstances that avert ovulation (use of oral contraceptives, multiparity, lactation) protect (by approximately 40\%) against the development of ovarian neoplasia. Ovarian cancer ranks fourth in cancerrelated deaths. It is the most common cause of fatality from a gynaecologic malignancy. Less than $25 \%$ of patients with advanced disease survive beyond 5 years (Runnebaum and Stickeler, 2001).

\section{Disease progression}

There are four basic stages of advancement in ovarian cancer (International Federation of Gynecology and Obstetrics). Stage I is defined by the formation of epithelial inclusion cysts that invade the ovarian cortex. Inclusion bodies are apparently formed when surface cells become entrapped within the ovarian wound created at ovulatory follicular rupture or during luteal resorption. Prognosis is good if the cyst has not ruptured and growth is limited internally to one (la) or both (Ib) ovaries. Malignant cells are extruded into the peritoneal cavity when an inclusion cyst ruptures (Ic). Peritoneal spread of cancer cells is the hallmark of stage II disease; this stage is subclassified depending upon the degree of pelvic extension (a: oviducts-uterus; b: other pelvic tissues) and generation of ascites fluid (c). Stage III is epitomized by the development of tumours involving one or both ovaries with peritoneal implants outside the pelvis (a: microscopic seeding; b and c: nodules $\leqslant$ or $\geqslant 2 \mathrm{~cm}$ in diameter, respectively) or positive retroperitoneal or inguinal nodes (c). Superficial liver metastasis and malignant extension to the small bowel or omentum are typical of invasive stage III. Stage IV disease is defined by ovarian growth with distant disseminated metastases to encompass pleural effusion and parenchymal liver (Hamilton, 1992).

Ovarian surface epithelial cells generally undergo metaplasia into a Mullerian duct derivative with morphological attributes akin to tubal, endometrial or endocervical epithelia: papillary serous, endometrioid or mucinous tumours, respectively. Clear-cell tumours, the most lethal subtype, are distinguished by epithelial nests in a fibromatous mesh. Unclassified epithelial growths exhibit a range of histological features. Borderline epithelial ovarian tumours, usually of the serous or mucinous types, have a low potential for growth or metastasis and present at an earlier stage than carcinomas (Feeley and Wells, 2001).

Early-stage ovarian cancer does not manifest conspicuous symptoms. Increased abdominal girth and pelvic pressure are indicative of the accumulation of ascites fluid and widespread metastases. Facial hair, thinning of the scalp, decreased breast size, and increased sex drive are sometimes evident, and these symptoms have been ascribed to androgen production by tumours (Tavassoli, 1994).

\section{Aetiology}

The sequence of events that leads to ovarian cancer is multifactorial and not adequately understood (Hamilton, 1992). It appears that the first step in tumorigenesis involves genomic disturbances to the ovarian surface epithelium that arise from ovulation (Fig. 3). Common epithelial ovarian 


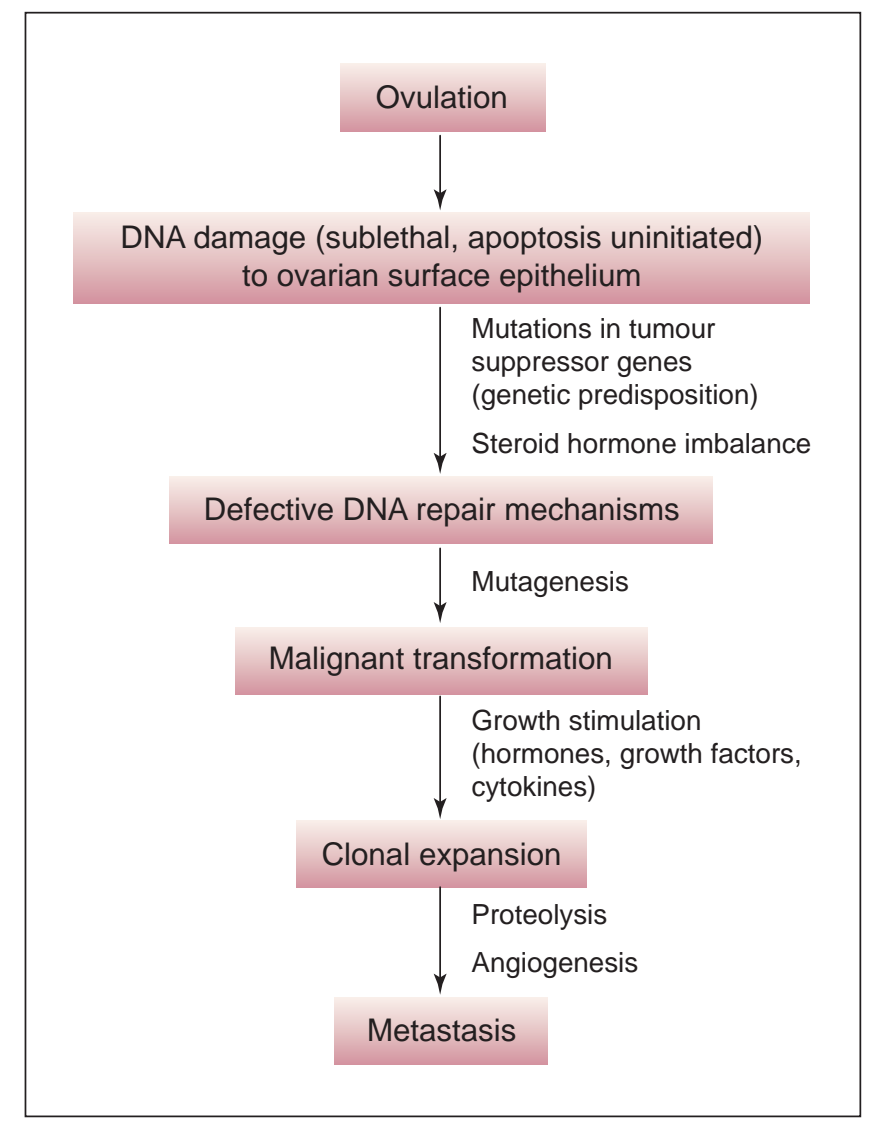

Fig. 3. Prospective role of ovulation in the chronology of ovarian carcinogenesis.

cancer is, in most cases, a unifocal disease ultimately arising by clonal expansion from a single transformed cell. Several aberrant steps are required to yield a malignant phenotype with a distinct growth advantage. Heredity is a determinant, especially in aggressive early-onset disease. Metastatic spread is protease-dependent. Ovarian cancer is generally considered to have some level of hormonal involvement. Local biochemical modulators can also affect behaviour of ovarian cancer cells. Some studies indicate that exposure to exogenous chemicals (for example, talc) and viruses (for example, mumps, which can cause premature ovarian failure) may increase susceptibility to ovarian cancer.

Ovulatory factor. The 'incessant ovulation hypothesis' of ovarian cancer was proposed by Fathalla (1971). The fact that anovulation prevents ovarian carcinoma lends incidental support to this hypothesis. Repeated ovulations, without long dormant periods, were suggested to cause malignant transformation of epithelium. In addition, ovulation, and therefore ovarian cancer, was more common in women than it was in most other species because females of other species are either pregnant or lactating for most of their reproductive lives. Accordingly, peritoneal carcinomatas occur in intensive egg-laying domestic hens (Fredrickson, 1987). There is some anecdotal evidence that borderline tumours can be provoked in women treated with fertility drugs (that is, menopausal or chorionic gonadotrophins and clomiphene citrate) used for superovulation in assisted reproduction programmes (Shoham, 1994).

Inflammatory mediators and reactive oxidants are generated during the ovulatory process (Ness and Cottreau, 1999). Persistence around the ovulatory wound of ovarian surface epithelial cells with DNA strand breaks and oxidative base (8-oxoguanine) damage (Murdoch et al., 2001) could be a determinant of carcinogenic onset. A genetically altered progenitor cell, with unrepaired DNA, but not committed to death, could give rise to a transformed phenotype that is propagated upon healing of the ovulatory wound. Oxoguanine is arguably the most important mutagenic lesion in DNA, in which a mispairing with adenine during replication causes a GC $\rightarrow$ TA transversion often detected in tumour cells (Grollman and Moriya, 1993). Fortunately, sublethal damage to DNA that is inflicted upon bystander ovarian surface epithelial cells at ovulation is normally reconciled by repair enzymes, including poly(ADP-ribose) polymerase and the base excision polymerase $\beta$, induced on a localized basis by progesterone of luteal derivation (Murdoch, 1998b, 2001). Progesterone also activates p53 expression and inhibits basal and oestrogen-stimulated proliferative responses in sheep ovarian surface epithelial cells (cell cycle arrest), thus affording the time required for DNA repair (Murdoch and Van Kirk, 2002).

Most human ovarian cancer cells exhibit aneuploidy (Berek et al., 1993). Induced chromosomal anomalies have been detected in rat and mouse ovarian surface epithelial cells subjected to repetitive subculturing, adding indirect support to the contention that mitotic reactions predicated by superfluous ovulation contribute to the pathogenesis of common ovarian cancer (Godwin et al., 1992; Roby et al., 2000). Anomalous ovarian cells sloughed during the ovulatory process may account for cases of disseminate disease in which the ovaries remain relatively uninvolved.

A simplified 'cumulative' ovulation model for ovarian cancer is not absolute. Protection is conferred by tubal ligation or hysterectomy in spite of uninterrupted ovulation; protection provided by a single gestation with breast feeding is far superior to the predicted benefit of those missed ovulations that would have occurred without pregnancy, and the advantage of short-term oral contraceptive use persists well after discontinuance (Scott, 1984). Moreover, overall disease occurrence has remained relatively constant over recent decades, despite the widespread application of ovulation-stimulating drugs (Glud et al., 1998).

Genetic predisposition. Three distinct hereditary conditions are associated with familial ovarian cancer: (1) dominant site-specific disease; (2) breast-ovarian cancer 
syndrome, with the clustering of breast and ovarian cancer cases in extended pedigrees; and (3) the Lynch type II cancer family syndrome, in which ovarian cancer is inherited together with non-polyposis colorectal and endometrial cancers (Auersperg et al., 2001). Congenital ovarian cancer occurs at an earlier age (35-40 years) than sporadic malignancy. Inheritance of deleted or malfunctional tumour suppressor genes (for example, those that overexpress competitive mutant forms of the growthinhibitory BRCA1/2, p53, DOC-2 or ARHI genes) is a possible basis for developing ovarian neoplasia as a result of ovulation (Aunoble et al., 2000). Because site-specific familial ovarian cancer is acquired on an autosomal chromosome, (unaffected) males can transmit predisposing genes to their daughters (Amos and Struewing, 1993).

Proteolytic enzymes. Members of the PA-plasmin cascade are of particular importance in the pathobiology of common epithelial ovarian cancer. Urokinase is the primary PA secreted by epithelial ovarian cancer cells. Phenotypes of ovarian tumours with high malignant and recurrent potentials accumulate uPA; it is liberated (with MMPs) from cells in membrane vesicles. The catabolic end-product of UPA action, plasmin, activates latent collagenases, which digest basement membranes and interstitial connective tissue matrices, providing an avenue for tumour cell invasiveness (Stack et al., 1998). Urokinase also facilitates tumour angiogenesis and growth (Rabbani, 1998). Immunoneutralization of UPA inhibits ovarian cancer cell invasion in vitro (Kobayashi et al., 1992) and the spread of human ovarian cancer in nude mice is reduced by antisense inhibition of uPA (Wilhelm et al., 1995).

Endocrine factors. Development of ovarian cancers has been related to excessive gonadotrophin production attributable to the onset of the menopause or premature ovarian failure. Receptors for gonadotrophic hormones have been detected in some cell lines and linked (via expression of growth factors) to increased proliferative and decreased apoptotic rates (Konishi et al., 1999).

Some ovarian tumours produce or can interconvert sex steroid hormones. Increased circulatory concentrations of progestogens, androgens or oestrogens have been correlated with tumour volume. It has been argued that steroid hormones emanate from sources outside the tumour, namely stromal ovarian and adrenal tissues. More than half of malignant ovarian tumours contain receptors for a steroid hormone (Rao and Slotman, 1991). It is evident that androgens and oestrogens do stimulate (again with the probable involvement of growth factors) growth-promoting genes in subsets of ovarian cancer cells. Postmenopausal oestrogen replacement therapy increases the risk of ovarian cancer mortality (Rodriguez et al., 2001), whereas progesterone appears to render protection (Risch, 1998). Secretion of uPA (shedding of exocytotic vesicles) and the invasiveness of SKOV-3 ovarian cancer cells were inhibited by progesterone (McDonnel and Murdoch, 2001).
Paracrine-autocrine factors. Growth factors and cytokines have been implicated in the progression of epithelial ovarian carcinomatosis. Epidermal growth factor, transforming growth factor $\alpha$ (TGF- $\alpha$ ), platelet-derived growth factor, basic fibroblast growth factor, hepatocyte growth factor, keratinocyte growth factor-kit ligand, insulin-like growth factor I, macrophage colony-stimulating factor, interleukin 1 (IL-1) and IL-6, TNF- $\alpha$, steroidogenesisinducing protein, and lysophosphatidic acid (LPA) promote loss of contact inhibition, cellular proliferation or protease secretion in vitro (TGF- $\beta$, interferons $\alpha$ and $\gamma$, high-dose TNF- $\alpha$, and GnRH were negative effectors) (Berchuck et al., 1993; Nash et al., 1999). Activation of dominant transforming mitogenic oncogenes (c-myc, $k$-ras, HER-2/neu) by growth factors and cytokines appears to be causally related to ovarian tumour formation (Aunoble et al., 2000; Auersperg et al., 2001). Vascular endothelial growth-permeability factor (VEGF) is secreted by ovarian cancer cells and has been related to ascites formation and metastasis (Auersperg et al., 2001).

\section{Diagnosis}

The main reason that ovarian cancer is so lethal is that it usually remains clinically silent until it becomes well established (that is, reaches stage III or IV). Detection of early-stage and recurrent disease continues to be a major problem. Diagnostic approaches have generally hinged on the principle that specific cell-surface antigens are shed from the tumour. The most thoroughly studied ovarian cancer antigen (CA) is the glycoprotein CA-125. Approximately $80 \%$ of patients with overt symptoms demonstrate an increase in immunoreactive serum CA-125. Individuals with mucinous tumours usually test negative. Problems with measuring CA-125 are the lack of specificity and insensitivity of tests. False positive results are common in pregnancy and occur in non-malignant premenopausal conditions of pelvic inflammatory disease, uterine fibroids, endometriosis, peritonitis, pancreatitis, renal failure and alcoholic hepatitis (Bast et al., 1998). Other ostensible indicators of ovarian cancer include mucins, soluble IL-2 receptor, VEGF, inhibin, galactosyltransferase, CA-72-4 and LPA (Menon and Jacobs, 2000).

Transvaginal sonography has been beneficial in identifying asymptomatic women with stage I ovarian cancer (Oram and Jeyarajah, 1994). Second-look laparotomy is sometimes used to assess disease status after completion of primary therapy (typically, cytoreductive surgery used in combination with platinum-containing drugs, alkylating agents or taxol) (Ozols, 1999), yet nearly 50\% of patients deemed negative relapse and die of diffuse disease (Podratz and Kinney, 1993).

The reality is that there is no combination of diagnostic tools available that have proven effective in reducing the mortality from ovarian cancer. Furthermore, it is not practical to screen the general population for a malignancy of relatively low incidence that necessitates surgical 
intervention. Applications of tests for early detection will probably be restricted principally to individuals designated as at high risk.

\section{Conclusion}

Recent research in sheep indicates that the ovarian surface epithelium plays an active role in the mechanism of ovulatory follicular rupture, and that damage to DNA imposed upon surface epithelial cells that survive the process of ovulation (and proliferate to repair the rupture wound), could be a predisposing (that is, potentially mutagenic) factor in ovarian cancer. Nevertheless, causeeffect associations between ovulatory disturbances to DNA and ovarian epithelial carcinogenesis have not been established. Future studies, to include additional species, are needed to resolve the pathophysiological relevance of the ovarian surface epithelium.

\section{References}

Key references are identified by asterisks.

Amos CI and Struewing JP (1993) Genetic epidemiology of epithelial ovarian cancer Cancer 71 566-572

*Auersperg N, Wong AST, Choi KC, Kang SK and Leung PCK (2001) Ovarian surface epithelium: biology, endocrinology, and pathology Endocrine Reviews 22 255-288

*Aunoble B, Sanches R, Didier E and Bignon YJ (2000) Major oncogenes and tumor suppressor genes involved in epithelial ovarian cancer International Journal of Oncology 16 567-576

Bast RC, Xu FJ, Yu YH, Barnhill S, Zhang Z and Mills GB (1998) CA 125: the past and future International Journal of Biological Markers 13 179-187

Berchuck A, Kohler MF, Boente M, Rodriguez GC, Whitaker RS and Bast RC (1993) Growth regulation and transformation of ovarian epithelium Cancer 71 545-551

Berek JS, Martinez-Maza O, Hamilton T, Trope C, Kaern J, Baak J and Rustin GJ (1993) Molecular and biological factors in the pathogenesis of ovarian cancer Annals of Oncology Supplement 4 3-16

Birkedal-Hansen H (1995) Proteolytic remodeling of extracellular matrix Current Opinion in Cell Biology 7 728-735

Bjersing L and Cajander S (1975) Ovulation and the role of the ovarian surface epithelium Experentia 31 605-608

Brännström M, Bonello N, Wang LJ and Norman RJ (1995) Effects of tumour necrosis factor $\alpha(\mathrm{TNF} \alpha)$ on ovulation in the rat ovary Reproduction, Fertility and Development 7 67-73

Byskov AG (1986) Differentiation of mammalian embryonic gonad Physiological Reviews 66 71-117

Cavender JL and Murdoch WJ (1988) Morphological studies of the microcirculatory system of periovulatory ovine follicles Biology of Reproduction 39 989-997

Colgin DC and Murdoch WJ (1997) Evidence for a role of the ovarian surface epithelium in the ovulatory mechanism of the sheep: secretion of urokinase-type plasminogen activator Animal Reproduction Science $\mathbf{4 7}$ 197-204

Danø K, Andreasen PA, Grøndahl-Hansen J, Kristensen P, Nielsen LS and Skriver L (1985) Plasminogen activators, tissue degradation, and cancer Advances in Cancer Research 44 139-266

Fathalla MF (1971) Incessant ovulation - a factor in ovarian neoplasia? Lancet 2163

Feeley KM and Wells M (2001) Precursor lesions of ovarian epithelial malignancy Histopathology 38 87-95

Fredrickson TN (1987) Ovarian tumors of the hen Environmental Health Perspectives 73 35-51

Ghahremani M, Foghi A and Dorrington JH (1999) Etiology of ovarian cancer: a proposed mechanism Medical Hypotheses 52 23-26
Glud E, Kjaer SK, Troisi R and Brinton LA (1998) Fertility drugs and ovarian cancer Epidemiologic Reviews 20 237-257

Godwin AK, Testa JR, Handel LM, Liu Z, Vanderveer LA, Tracey PA and Hamilton TC (1992) Spontaneous transformation of rat ovarian surface epithelial cells: association with cytogenetic changes and implications of repeated ovulation in the etiology of ovarian cancer Journal of the National Cancer Institute 84 592-601

Gottsch ML, Van Kirk EA and Murdoch WJ (2000) Tumour necrosis factor $\alpha$ upregulates matrix metalloproteinase-2 activity in periovulatory ovine follicles: metamorphic and endocrine implications Reproduction, Fertility and Development 12 75-80

Grollman AP and Moriya M (1993) Mutagenesis by 8-oxoguanine: an enemy within Trends in Genetics 9 246-249

Hägglund A-C, Ny A, Liu K and Ny T (1996) Coordinated and cell-specific induction of both physiological plasminogen activators creates functionally redundant mechanisms for plasmin formation during ovulation Endocrinology 137 5671-5677

Hall JA, Meisterling EM, Benoit AM, Cooper DA, Coleman DA, Lerner SP, Lewis PE and Dailey RA (1993) Factors contributing to the formation of experimentally induced ovarian cysts in prepubertal gilts Domestic Animal Endocrinology 10 141-155

Hamilton TC (1992) Ovarian cancer, biology Current Problems in Cancer 16 5-57

Johnson ML, Murdoch J, Van Kirk EA, Kaltenbach JE and Murdoch WJ (1999) Tumor necrosis factor $\alpha$ regulates collagenolytic activity in preovulatory ovine follicles: relationship to cytokine secretion by the oocyte-cumulus cell complex Biology of Reproduction $\mathbf{6 1}$ $1581-1585$

Kobayashi H, Ohi H, Sugimura M, Shionhara H, Fujii T and Terao T (1992) Inhibition of in vitro ovarian cancer cell invasion by modulation of urokinase-type plasminogen activator and cathepsin B Cancer Research $523610-3614$

Konishi I, Kuroda H and Mandai M (1999) Gonadotropins and development of ovarian cancer Oncology Supplement 57 45-48

Larrick JW and Wright SC (1990) Cytotoxic mechanism of tumor necrosis factor $\alpha$ FASEB Journal 4 3215-3223

Luck MR (1994) The gonadal extracellular matrix Oxford Reviews of Reproductive Biology 16 34-85

McDonnel AC and Murdoch WJ (2001) High-dose progesterone inhibition of urokinase secretion and invasive activity by SKOV-3 ovarian carcinoma cells: evidence for a receptor-independent nongenomic effect on the plasma membrane Journal of Steroid Biochemistry and Molecular Biology 78 185-191

Menon U and Jacobs IJ (2000) Recent developments in ovarian cancer screening Current Opinion in Obstetrics and Gynecology 12 39-42

Murdoch WJ (1994) Ovarian surface epithelium during ovulatory and anovulatory ovine estrous cycles Anatomical Record 240 322-326

*Murdoch WJ (1998a) Regulation of collagenolysis and cell death by plasmin within the formative stigma of preovulatory ovine follicles Journal of Reproduction and Fertility 113 331-336

Murdoch WJ (1998b) Perturbation of sheep ovarian surface epithelial cells by ovulation: evidence for roles of progesterone and poly(ADP-ribose) polymerase in the restoration of DNA integrity Journal of Endocrinology 156 503-508

Murdoch WJ and Lund SA (1999) Prostaglandin-independent anovulatory mechanism of indomethacin action: inhibition of tumor necrosis factor $\alpha$-induced sheep ovarian cell apoptosis Biology of Reproduction $\mathbf{6 1}$ 1655-1659

Murdoch WJ and McCormick RJ (1992) Enhanced degradation of collagen in apical versus basal wall of ovulatory ovine follicle American Journal of Physiology $263 \mathrm{E} 221-225$

Murdoch WJ and Van Kirk EA (2002) Steroid hormonal regulation of proliferative, p53 tumor suppressor, and apoptotic responses of sheep ovarian surface epithelial cells Molecular and Cellular Endocrinology $18661-67$

Murdoch WJ, Colgin DC and Ellis JA (1997) Role of tumor necrosis factor $\alpha$ in the ovulatory mechanism of ewes Journal of Animal Science $\mathbf{7 5}$ 1601-1605

Murdoch J, Van Kirk EA and Murdoch WJ (1999a) Hormonal control of 
urokinase plasminogen activator secretion by sheep ovarian surface epithelial cells Biology of Reproduction 61 1487-1491

Murdoch WJ, Van Kirk EA and Murdoch J (1999b) Plasmin cleaves tumor necrosis factor $\alpha$ exodomain from sheep follicular endothelium: implication in the ovulatory process Biology of Reproduction $\mathbf{6 0}$ 1166-1171

Murdoch WJ, Wilken C and Young DA (1999c) Sequence of apoptosis and inflammatory necrosis within the formative ovulatory site of sheep follicles Journal of Reproduction and Fertility 117 325-329

*Murdoch WJ, Townsend RS and McDonnel AC (2001) Ovulation-induced DNA damage in ovarian surface epithelial cells of ewes: prospective regulatory mechanisms of repair/survival and apoptosis Biology of Reproduction 65 1417-1424

Nash MA, Ferrandina G, Gordinier M, Loercher A and Freedman RS (1999) The role of cytokines in both the normal and malignant ovary EndocrineRelated Cancer 6 93-107

Ness RB and Cottreau C (1999) Possible role of ovarian epithelial inflammation in ovarian cancer Journal of the National Cancer Institute 91 1459-1467

Oram DH and Jeyarajah AR (1994) The role of ultrasound and tumour markers in the early detection of ovarian cancer British Journal of Obstetrics and Gynaecology 101 939-945

Ozols RF (1999) Chemotherapy for ovarian cancer Seminars in Oncology Supplement 26 34-40

Peng X-R, Hsueh AJW and Ny T (1993) Transient and cell-specific expression of tissue-type plasminogen activator and plasminogenactivator-inhibitor type 1 results in controlled and directed proteolysis during gonadotropin-induced ovulation European Journal of Biochemistry 214 147-156

Podratz KC and Kinney WK (1993) Second-look operation in ovarian cancer Cancer $\mathbf{7 1}$ 1551-1558

Rabbani SA (1998) Metalloproteinases and urokinase in angiogenesis and tumor progression In Vivo 12 135-142

* Rao BR and Slotman BJ (1991) Endocrine factors in common epithelial ovarian cancer Endocrine Reviews 12 14-26

Rawson JMR and Espey LL (1977) Concentration of electron dense granules in the rabbit ovarian surface epithelium during ovulation Biology of Reproduction 17 561-566

Risch HA (1998) Hormonal etiology of epithelial ovarian cancer, with a hypothesis concerning the role of androgens and progesterone Journal of the National Cancer Institute 90 1774-1786

Roby KF, Taylor CC, Sweetwood JS, Cheng Y, Pace JL, Tawfik O, Persons DL, Smith PG and Terranova PF (2000) Development of a syngeneic mouse model for events related to ovarian cancer Carcinogenesis 21 585-591

Rodriguez C, Patel AV, Calle EE, Jacob EJ and Thun MJ (2001) Estrogen replacement therapy and ovarian cancer mortality in a large prospective study of US women Journal of the American Medical Association 285 1460-1465

Runnebaum IB and Stickeler E (2001) Epidemiological and molecular aspects of ovarian cancer risk Journal of Cancer Research and Clinical Oncology 127 73-79

Schuetz AW and Lessman C (1982) Evidence for follicle wall involvement in ovulation and progesterone production by frog (Rana pipiens) follicles in vitro. Differentiation 22 79-84

Scott JS (1984) How to induce ovarian cancer: and how not to British Medical Journal $289781-782$

Shoham Z (1994) Epidemiology, etiology, and fertility drugs in ovarian epithelial carcinoma: where are we today? Fertility and Sterility 62 433-448

Smith MF, Mclntush EW, Ricke WA, Kojima FN and Smith GW (1999) Regulation of ovarian matrix remodelling by metalloproteinases and their tissue inhibitors: effects on follicular development, ovulation and luteal function Journal of Reproduction and Fertility Supplement $\mathbf{5 4}$ 367-381

Smokovitis A, Kokolis $\mathbf{N}$ and Alexaki-Tzivanidou $\mathbf{E}$ (1988) The plasminogen activator activity is markedly increased mainly at the area of the rupture of the follicular wall at the time of ovulation Animal Reproduction Science 16 285-294

*Stack MS, Ellerbroek SM and Fishman DA (1998) The role of proteolytic enzymes in the pathology of epithelial ovarian carcinoma International Journal of Oncology 12 569-576

Tavassoli FA (1994) Ovarian tumors with functioning manifestations Endocrine Pathology 5 137-148

Terranova PF (1997) Potential roles of tumor necrosis factor $\alpha$ in follicula development, ovulation, and the life span of the corpus luteum Domestic Animal Endocrinology 14 1-15

Tsafriri A and Reich R (1991) Plasminogen activators in the preovulatory follicle: role in ovulation. In Plasminogen Activators: From Cloning to Therapy pp 81-93 Eds R Abbate et al. Raven Press, New York

Walt ML, Stabenfeldt GH, Hughes JP, Neely DP and Bradbury R (1979) Development of the equine ovary and ovulation fossa Journal of Reproduction and Fertility Supplement 27 471-477

Warzocha K and Salles G (1998) The tumor necrosis factor signaling complex: choosing a pathway toward cell death or cell proliferation Leukemia Lymphoma 29 81-92

Wilhelm O, Schmitt M, Hohl S, Senekowitsch R and Graeff H (1995 Antisense inhibition of urokinase reduces spread of human ovarian cancer in mice Clinical and Experimental Metastasis 13 296-302

Woessner JF, Morioka N, Zhu C, Mukaida T, Butler T and LeMaire WJ (1989) Connective tissue breakdown in ovulation Steroids 54 491-499 